\title{
Uterine Ligament Adenosarcoma
}

National Cancer Institute

\section{Source}

National Cancer Institute. Uterine Ligament Adenosarcoma. NCI Thesaurus. Code C102570.

An extremely rare adenosarcoma that arises from the uterine ligament. 